\title{
3D Oxalate-Based Coordination Polymers: Relationship between Structure, Magnetism and Color, studied by High-Field ESR Spectroscopy ${ }^{\text {th }}$
}

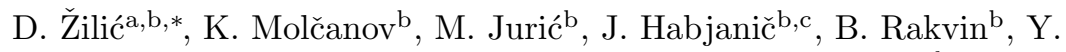 \\ Krupskaya $^{\mathrm{a}}$, V. Kataev ${ }^{\mathrm{a}}$, S. Wurmehla ${ }^{\mathrm{a}}$, B. Büchner ${ }^{\mathrm{a}, \mathrm{d}}$ \\ ${ }^{a}$ Institute for Solid State Research, IFW Dresden, Helmholtzstrasse 20, D-01069 Dresden, \\ Germany \\ ${ }^{b}$ Ruđer Bošković Institute, Bijenička cesta 54, 10000 Zagreb \\ ${ }^{c}$ University of Zurich, Rämistrasse 71, CH-8006 Zürich, Switzerland \\ ${ }^{d}$ Institute for Solid State Physics, Technical University Dresden, D-01062 Dresden, \\ Germany
}

\begin{abstract}
The detailed high field electron spin resonance (HF-ESR) study of three 3D oxalate-based coordination polymers with antiferromagnetic ordering below 13 $\mathrm{K}$, supported by crystallographic, photoluminescence (PL), elemental (EDX) and X-band ESR analysis, is presented. Two of the investigated compounds are described by the same chemical formula $\left\{\left[\mathrm{Cu}(\text { bpy })_{3}\right]\left[\mathrm{Mn}_{2}\left(\mathrm{C}_{2} \mathrm{O}_{4}\right)_{3}\right] \cdot \mathrm{H}_{2} \mathrm{O}\right\}_{n}$, both exhibit PL but have different colors: green-blue and red-pink (CuMn2Green and CuMn2-Red, respectively) while $\left\{\left[\mathrm{Co}(\text { bpy })_{3}\right]\left[\mathrm{Mn}_{2}\left(\mathrm{C}_{2} \mathrm{O}_{4}\right)_{3}\right] \cdot \mathrm{H}_{2} \mathrm{O}\right\}_{n}$ is yellow (CoMn2-Yellow) without PL. Despite different colors of CuMn2-Green and CuMn2-Red, X-ray diffraction, EDX, as well as X-band ESR, could not reveal any difference between these compounds. Therefore, detailed temperature dependent multifrequency HF-ESR experiments were performed. Owing to very high resolution of HF-ESR, two different $\mathrm{Mn}$ (II) centers in CuMn2-Green and CoMn2-Yellow compounds could be resolved, while in CuMn2-Red only one
\end{abstract}

\footnotetext{
${ }^{\hbar}$ Electronic Supplementary Information (ESI) available: [CIF file and crystallographic data of CuMn2-Red; SEM pictures, EDX spectra and Mn:Cu EDX ratios for CuMn2-Green and CuMn2-Red; PL spectra of CuMn2-Green, CuMn2-Red and CoMn2-Yellow; X-band ESR study with CuMn2-Red and CoMn2-Yellow spectra and simulation for CoMn2-Yellow. CCDC 1504116]. For ESI and crystallographic data in CIF or other electronic format see DOI.....

* Corresponding author

Email address: dzilic@irb.hr (D. Žilić)
} 
$\mathrm{Mn}$ (II) center is detected. A possible difference in the ligand coordination of $\mathrm{Mn}$ (II) ions in the oxalate-networks of CuMn2-Green and CuMn2-Red, that gives rise to slightly different $g$-factors, is discussed with respect to the different colors of these compounds.

Keywords: ESR, high field, high frequency, coordination polymer, $\mathrm{Mn}(\mathrm{II})$

2016 MSC: 00-01, 99-00

\section{Introduction}

Coordination polymers (CPs) and metal-organic frameworks (MOFs) are among the most prolific research areas of inorganic chemistry and crystal engineering in the last two decades. 1, 2] They could be identified as a class of 5 porous polymeric materials, consisting of metal ions linked together by organic bridging ligands. 1, 3] Due to the variety of metal ions and ligands, coordination geometry, guests inside the pores and supramolecular structures, an enormous number of CPs with a broad range of structural, catalytic, electrical, magnetic and optical properties has been reported. 3, 4, 5, 6, 7, 8,

Oxalate-based CPs have drawn considerable attention in the field of moleculebased magnets due to high efficiency of oxalate bridge $\mathrm{C}_{2} \mathrm{O}_{4}{ }^{2-}$ in transmitting the exchange interaction between metal ions. 9. The tris(oxalato)metalate anions $\left[\mathrm{M}\left(\mathrm{C}_{2} \mathrm{O}_{4}\right)_{3}\right]^{3-}(\mathrm{M}=$ trivalent metal $)$ have been widely used as ligands toward metal ions in the preparation of two- (2D) and three-dimensional (3D) networks of the general formula $\left[\mathrm{M}_{a}{ }^{I I / I I I} \mathrm{M}_{b}{ }^{I / I I}\left(\mathrm{C}_{2} \mathrm{O}_{4}\right)_{3}\right]_{n}{ }^{2 n-/ n-}$. New multifunctional systems are composed of these polymeric oxalate anionic networks, that exhibit magnetic properties (ferro-, ferri- or canted antiferromagnetism) and a bulky counterion, which templates the dimensionality of these networks and could add a second physical property of interest. [10, 11, 12, 13.

20 A special position have new materials that combine magnetism and luminiscence, due to desire to control magnetic phase transition of molecule-based magnetic compounds under optical stimuli. 14 Besides searching for multifunctional materials and their applications in optoelectronics, luminescent molecule-based 
magnets are interesting also with respect to the fundamental understanding of magnetism. Namely, most of the transition metal ions are not emissive due to the nonradiative relaxation through interactions with low-lying excited states, that makes synthesis of emissive magnets very difficult. [15, 16]

Besides progress in chemical synthesis, a better understanding of structural and magnetic correlations is achieved due to the progress in experimental techniques in physics. Electron spin or paramagnetic resonance (ESR or EPR) spectroscopy provides insights into local properties of paramagnetic centers and microscopic picture of the interactions. However, commercially used ESR spectrometers, using a few selected frequencies below $100 \mathrm{GHz}$, do not always meet necessary requirements for investigation of transition metal complexes with spin $35 \quad S>1 / 2$. To obtain the spin-Hamiltonian parameters, g-tensor, D-tensor (zerofield splitting) and A-tensor (hyperfine splitting), for the complexes with spin $S>1 / 2$, it is necessary to employ ESR spectrometers at high (sub-THz) frequencies performing continuous magnetic field sweeps over a broad range (highfield/high-frequency ESR, HF-ESR). [17, 18, 19, 20, 21] In addition to possibility to study electron spin systems with large zero-field splitting and to detect very broad absorption lines, HF-ESR also provides a much better resolution of $g$ values.

In this work, detailed investigation of three 3D oxalate-based CPs with antiferromagnetic (AFM) ordering below $13 \mathrm{~K}$, described by general formula ${ }_{45}\left\{\left[\mathrm{X}(\mathrm{bpy})_{3}\right]\left[\mathrm{Mn}_{2}\left(\mathrm{C}_{2} \mathrm{O}_{4}\right)_{3}\right] \cdot \mathrm{H}_{2} \mathrm{O}\right\}_{n}$, where $\mathrm{X}=\mathrm{Cu}(\mathrm{II})$ or $\mathrm{Co}(\mathrm{II})$, is presented. Two of the investigated compounds are described by the same chemical formula $\left\{\left[\mathrm{Cu}(\text { bpy })_{3}\right]\left[\mathrm{Mn}_{2}\left(\mathrm{C}_{2} \mathrm{O}_{4}\right)_{3}\right] \cdot \mathrm{H}_{2} \mathrm{O}\right\}_{n}$, both show photoluminescence (PL), but have different colors: green-blue (CuMn2-Green) and red-pink (CuMn2-Red). The third sample with the chemical formula $\left\{\left[\mathrm{Co}(\mathrm{bpy})_{3}\right]\left[\mathrm{Mn}_{2}\left(\mathrm{C}_{2} \mathrm{O}_{4}\right)_{3}\right] \cdot \mathrm{H}_{2} \mathrm{O}\right\}_{n}$ has 50 yellow color without PL (CoMn2-Yellow). Here, synthesis and crystal structure of CuMn2-Red together with elemental analysis (EDX) of CuMn2-Green and CuMn2-Red are presented. Despite the different colors, X-ray diffraction, as well as X-band ESR spectroscopy, could not reveal any difference between CuMn2-Green and CuMn2-Red compounds. CoMn2-Yellow complex was used 
here as a reference sample. Moreover EDX analysis has shown approximately the same manganese vs. copper ratios in CuMn2-Green and CuMn2-Red. To analyze other possible differences between these two compounds, which could cause the difference in color, we have performed temperature-dependent multifrequency HF-ESR experiments (at frequencies 83, 166, 249 and $332 \mathrm{GHz}$ ) in

${ }_{60}$ high magnetic fields (up to $16 \mathrm{~T}$ ) together with the simulation of the ESR spectra. HF-ESR spectroscopy has shown that while CuMn2-Red sample has only one $\mathrm{Mn}(\mathrm{II})$ line at $g_{1}$ value, CuMn2-Green and CoMn2-Yellow samples show splitting of $\mathrm{Mn}(\mathrm{II})$ line into 2 components (characterized by $g_{1}$ and $g_{2}$ values). The observation of two $g$ values points to the existence of two slightly different $\mathrm{Mn}(\mathrm{II})$ centers in CuMn2-Green and CoMn2-Yellow. This result indicates different ligand coordinations of $\mathrm{Mn}(\mathrm{II})$ ions in CuMn2-Green and CuMn2-Red compounds which can account for different colors of these compounds.

\section{Experimental}

\subsection{Materials and Physical Measurements}

The chemicals were purchased from commercial sources and used without further purification. The starting species $\mathrm{K}_{3}\left[\mathrm{Mn}\left(\mathrm{C}_{2} \mathrm{O}_{4}\right)_{3}\right] \cdot 3 \mathrm{H}_{2} \mathrm{O}$ and $\left[\mathrm{Cu}(\mathrm{bpy})_{3}\right]-$ $\mathrm{Cl}_{2} \cdot 6 \mathrm{H}_{2} \mathrm{O}$ were prepared according to the method described in the literature. 22 , 23. The infrared spectra were recorded in the $4000-350 \mathrm{~cm}^{-1}$ region with the samples as $\mathrm{KBr}$ pellets with a Bruker Alpha FTIR spectrometer. The qual-

75 ity of the single crystals was examined with a scanning electron microscope (SEM, XL30 Philips, IN400) equipped with an electron microprobe analyzer for semiquantitative elemental analysis using the energy dispersive X-ray (EDX) mode. The photoluminescence spectra are recorded using laser radiation at $\lambda_{\text {exc }}=405 \mathrm{~nm}$ with power of $5 \mathrm{~mW}$.

\subsection{Preparation of CuMn2-Red}

Preparation of CuMn2-Green and CoMn2-Yellow was described in Ref. [11] and Ref. [10], respectively. Here we describe the preparation of CuMn2-Red. 
After mixing an aqueous solution $(3 \mathrm{~mL})$ of $\mathrm{K}_{3}\left[\mathrm{Mn}\left(\mathrm{C}_{2} \mathrm{O}_{4}\right)_{3}\right] \cdot 3 \mathrm{H}_{2} \mathrm{O}(49.0 \mathrm{mg}$; $0.1 \mathrm{mmol})$ with an aqueous solution $(5 \mathrm{~mL})$ of $\left[\mathrm{Cu}(\mathrm{bpy})_{3}\right] \mathrm{Cl}_{2} \cdot 6 \mathrm{H}_{2} \mathrm{O}(71.1 \mathrm{mg}$; diately formed. It was removed by filtration and the clear blue solution was layered with acetonitrile $(5 \mathrm{ml})$ in a test tube. Reddish octahedral crystals were appeared after more than one month (yield: $10 \%$. IR data $\left(\mathrm{KBr}, \mathrm{cm}^{-1}\right): 3443$ (w, br), 3101 (w), 3075 (w), 2931 (w),1655 (m), 1631 (s), 1607 (vs), 1487 (m), 1155 (m), 1099 (w), 1062 (w), 1017 (m), 984 (w), 919 (m), 797 (s), 778 (s), 736 (m), 651 (m), $630(\mathrm{w}), 568(\mathrm{w}), 489(\mathrm{~m}), 441(\mathrm{w}), 421(\mathrm{~m}), 384(\mathrm{~m}))$.

\subsection{Single-crystal $X$-ray study of CuMn2-Red}

The single-crystal X-ray diffraction measurement of CuMn2-Red was per$\mathrm{Cu}-\mathrm{K} \alpha$ radiation $(\lambda=1.54179 \AA$, microfocus tube, CCD detector $)$ at room temperature (293(2) K). The Friedel pairs were measured in order to unambiguously establish absolute configurations of octahedral stereogenic centres. The space group, absolute configuration and absolute structure were easily determined using $R$ values and the Flack parameter, which was $0.004(5)$. Therefore, the correct absolute configurations of the octahedral metal centers are $\Delta$ and the metal-oxalate helices are $M$. Attempt of refinement with $\Lambda$ enantiomers $(P$ helices) yielded $R$ value of 0.34 and meaningless Flack parameter of $0.7(1)$; refinement of the same enantiomers in the enantiomeric space group $P 4_{3} 32$ yielded $R$ of 0.104 and the Flack parameter of 0.96(4) indicated wrong absolute structure. The program package CrysAlis $\mathrm{PRO} 24]$ was used for data reduction. The structure was solved with SHELXS97 25] and refined with SHELXL-2013. 25] A model was refined by a full-matrix least-squares refinement; all non-hydrogen atoms were refined anisotropically. The hydrogen atoms were modelled as riding entities by using the AFIX command in SHELXL-2013. 25]. Molecular geometry calculations were performed by PLATON 26] and molecular graphics were prepared using ORTEP-3 27] and CCDC-Mercury (version 3.8).28] The crystal 
data, experimental conditions and final refinement parameters for the structure reported are summarized in Suppl. Table 1 in Electronic Supplementary Information (ESI).

\subsection{ESR spectroscopy}

$\mathrm{X}$-band electron spin resonance (ESR) measurements were performed using Bruker Elexsys $580 \mathrm{FT} / \mathrm{CW}$ spectrometer (CuMn2-Red sample) and Bruker EMX spectrometer (CoMn2-Yellow sample). The results were obtained from room temperature down to that of liquid helium, at the microwave frequency around $9.6 \mathrm{GHz}$, with the magnetic field modulation amplitude of $0.3 \mathrm{mT}$ at $100 \mathrm{kHz}$.

HF-ESR measurements were performed on powder samples using a homemade spectrometer based on Millimeter Vector Network Analyzer (AB Millimetre, Paris) that generates millimeter and submillimeter microwaves and performs phase locked detection of a signal. The spectrometer uses superconducting magnetocryostat from Oxford Instruments Ltd, with magnetic fields up to 16 T. For details see Ref. [29]. The HF-ESR spectra were recorded at four frequencies: 83, 166, 249 and $332 \mathrm{GHz}$, in the temperature range from $4 \mathrm{~K}$ up to $150 \mathrm{~K}$.

\section{Results}

\subsection{Characterization}

The CuMn2-Red crystals were obtained by the layering technique i.e. by slow liquid diffusion. 30] They are air-stable and not soluble in water and common organic solvents. Due to the reduction of $\mathrm{Mn}$ (III) in slightly acidic solutions, oxalate anionic network contains $\mathrm{Mn}(\mathrm{II})$. 22 .

Absorption bands in the IR spectrum of CuMn2-Red can be attributed to the vibrations of the bis(bidentate) bridging oxalate groups, coordinated 2,2'bipyridine ligands, and water molecules. The broad band of weak intensity with a maximum of around $3443 \mathrm{~cm}^{-1}$ is in agreement with the presence of water molecules. The absorption bands corresponding to the bridging oxalate ligand 
are located at 1655 and $1631 \mathrm{~cm}^{-1}\left[\nu_{a s}(\mathrm{CO})\right], 1359$ and $1309 \mathrm{~cm}^{-1}\left[\nu_{s}(\mathrm{CO})\right]$, and $797 \mathrm{~cm}^{-1}[\delta(\mathrm{OCO})]$. Other absorption bands in the spectra originate from different vibrations of coordinated 2,2'-bipyridine molecules. 31.

Suppl. Fig. 1 shows SEM pictures of a CuMn2-Green and CuMn2-Red single crystals while Suppl. Fig. 2 shows typical EDX spectra. Manganese vs. copper atomic ratios are presented in Suppl. Table 2 and it could be seen that the average $\mathrm{Mn}: \mathrm{Cu}$ atomic ratio, measured by EDX, is $2.02 \pm 0.04$ and $1.97 \pm 0.02$ for CuMn2-Green and CuMn2-Red samples, respectively.

The photoluminescence spectra of the CuMn2-Green, CuMn2-Red and CoMn2Yellow were recorded at room temperature and are available in the SI (Suppl. Fig. 3). At an excitation irradiation of $405 \mathrm{~nm}, \mathrm{CoMn} 2$-Yellow shows no luminescence while CuMn2-Green and CuMn2-Red show luminescence with a maxima at 462 and $490 \mathrm{~nm}$.

\subsection{Molecular and crystal structure of CuMn2-Red}

The crystal structure of $\Lambda$ enantiomer of CuMn2-Green has already been published in Ref. [11] while $\Delta$ enantiomer structure of CuMn2-Red crystal is presented in this study. Namely, CuMn2-Red compound, as well as CuMn2Green, crystallized as racemic conglomerates, containing enantiomeric crystals belonging to the space groups, $P 4_{1} 32$ and $P 4_{3} 32$. CuMn2-Red is isostructural to previously prepared $\left\{\left[\mathrm{Co}(\text { bpy })_{3}\right]\left[\mathrm{Mn}_{2}\left(\mathrm{C}_{2} \mathrm{O}_{4}\right)_{3}\right] \cdot \mathrm{H}_{2} \mathrm{O}\right\}_{n}$ (CoMn2-Yellow). [10] The unit cell parameter $(a)$ is $15.6685(1)$ and $15.6547(2) \AA$, so the unit cell volume is 3846.65(4) and 3836.49(15) $\AA^{3}$, for CoMn2-Yellow and CuMn2-Red, respectively. Atom positions are identical within experimental error, so the structure of CuMn2-Red compound can be refined using atomic coordinates from CoMn2-Yellow compound. CuMn2-Red comprises tris-chelated cations $\left[\mathrm{Cu}(\text { bpy) }]_{3}\right]^{2+}$ (Fig. 1 (a)) and polymeric $\left\{\mathrm{Mn}_{2}\left(\mathrm{C}_{2} \mathrm{O}_{4}\right)_{3}\right\}_{n} 2^{n-}$ anion (Fig. 1 (b)), and an uncoordinated water molecule disordered about a 3 -fold axis. $\mathrm{Cu}$ and $\mathrm{Mn}$ atoms are located in special positions: the $\mathrm{Cu}$ at an intersection of a 3-fold and three 2-fold axes and Mn at a threefold axis, coordination of both metal centres 

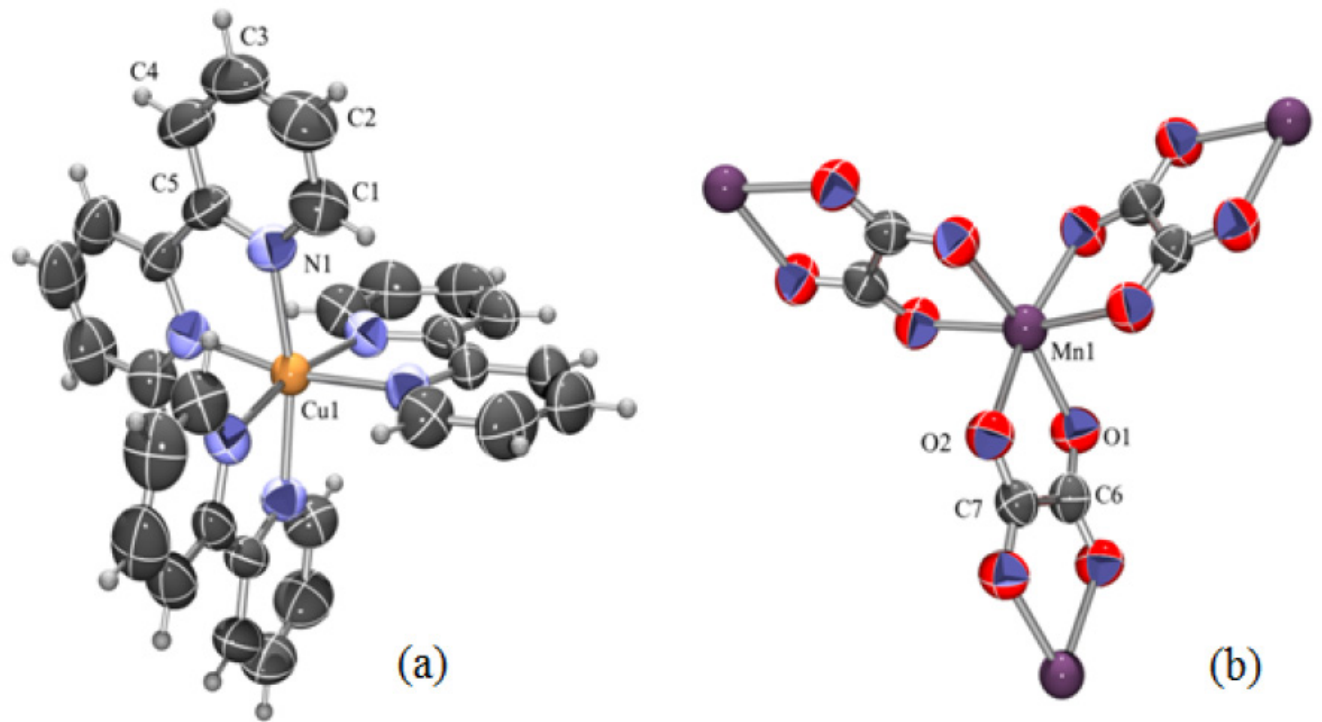

Figure 1: Molecular structure of: (a) tris-chelated $\left[\mathrm{Cu}(\mathrm{bpy})_{3}\right]^{2+}$ cation and (b) polymeric $\left\{\mathrm{Mn}_{2}\left(\mathrm{C}_{2} \mathrm{O}_{4}\right)_{3}\right\}_{n}^{2 n-}$ anion in CuMn2-Red with atom numbering scheme. Octahedrally coordinated metal atoms have a $\Delta$ configuration. Atomic displacement ellipsoids are drawn for the probability of $50 \%$ and the hydrogen atoms are depicted as spheres of arbitrary radii.

is clathrate-like, with a well-known porous 3D 3-connected (10,3) 32] oxalatebased $\left\{\mathrm{Mn}_{2}\left(\mathrm{C}_{2} \mathrm{O}_{4}\right)_{3}\right\}_{n}{ }^{2 n-}$ network (Fig. 2); the complex $\left[\mathrm{Cu}(\text { bpy })_{3}\right]^{2+}$ cations are located inside its large decagonal voids (Fig. 2). Absolute configuration of the crystal used in this study was established: the space group is $P 4_{1} 32$ and both octahedral stereogenic centers in compound $\left\{\left[\mathrm{Cu}(\text { bpy })_{3}\right]\left[\mathrm{Mn}_{2}\left(\mathrm{C}_{2} \mathrm{O}_{4}\right)_{3}\right] \cdot \mathrm{H}_{2} \mathrm{O}\right\}_{n}$ have a $\Delta$ configuration while the metal-oxalate anionic network consists of a $M$ helices. [10, 33]

\subsection{X-band ESR spectroscopy}

X-band ESR spectroscopy has not revealed any significant difference between CuMn2-Green and CuMn2-Red complexes and therefore the results are presented in SI. 
Table 1: Geometric parameters $\left(\AA,^{\circ}\right)$ of the metals coordination sphere in CuMn2-Red.

\begin{tabular}{cccc}
\hline $\mathrm{Cu} 1-\mathrm{N} 1$ & $2.121(3)$ & $\mathrm{N}^{i}-\mathrm{Cu} 1-\mathrm{N} 1^{i i}$ & $168.04(17)$ \\
$\mathrm{Mn} 1-\mathrm{O} 1$ & $2.161(3)$ & $\mathrm{O} 1-\mathrm{Mn} 1-\mathrm{O} 1^{i i i}$ & $99.19(9)$ \\
$\mathrm{Mn} 1-\mathrm{O} 2$ & $2.171(3)$ & $\mathrm{O} 1-\mathrm{Mn} 1-\mathrm{O} 2^{i v}$ & $93.53(11)$ \\
$\mathrm{N} 1-\mathrm{Cu} 1-\mathrm{N} 1^{i}$ & $95.17(17)$ & $\mathrm{O} 1-\mathrm{Mn} 1-\mathrm{O} 2$ & $77.47(9)$ \\
$\mathrm{N} 1-\mathrm{Cu} 1-\mathrm{N} 1^{i i}$ & $94.14(11)$ & $\mathrm{O} 1-\mathrm{Mn} 1-\mathrm{O} 2^{i v}$ & $167.23(10)$ \\
$\mathrm{N} 1-\mathrm{Cu} 1-\mathrm{N} 1^{i i i}$ & $77.71(17)$ & $\mathrm{O} 1-\mathrm{Mn} 1-\mathrm{O} 2^{i i i}$ & $90.36(11)$
\end{tabular}

Symmetry operators: (i) $-x+3 / 4,-z+3 / 4,-y+3 / 4$; (ii) $-z+1 / 2,-x+$ $1,-y+1 / 2 ;$ (iii) $z+1 / 4,-y+5 / 4, x-1 / 4$; (iv) $-y+1, z+1 / 2,-x+1 / 2$.

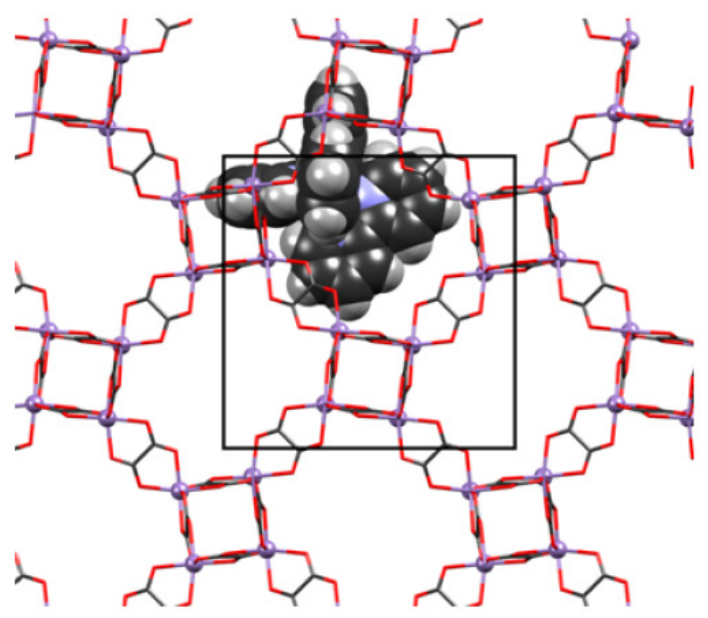

Figure 2: Crystal packing of CuMn2-Red viewed in the direction [111], showing a 3D $(10,3)$ oxalate-based anionic $\left\{\mathrm{Mn}_{2}\left(\mathrm{C}_{2} \mathrm{O}_{4}\right)_{3}\right\}_{n}{ }^{2 n-}$ network with $\left[\mathrm{Cu}(\text { bpy })_{3}\right]^{2+}$ cations occupying decagonal voids. 


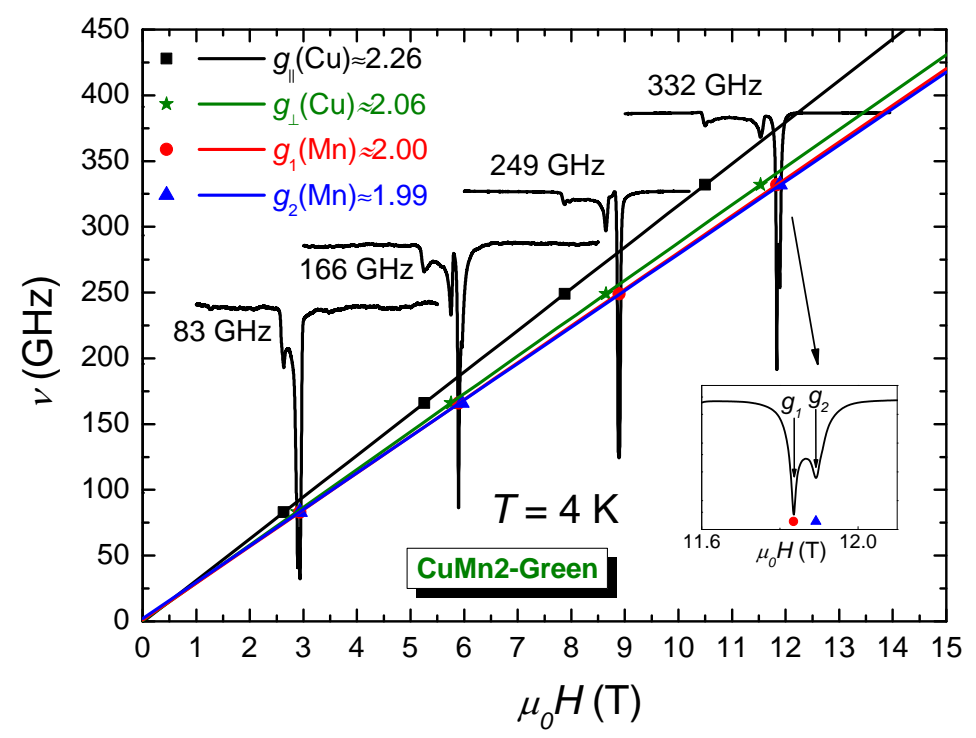

Figure 3: Frequency $\nu$ vs. resonance magnetic field $\mu_{0} H$ dependence (symbols), together with corresponding spectra, at $T=4 \mathrm{~K}$. Solid lines are linear fits to the experimental data (symbols). Inset: Enlarged central part of the spectra at $332 \mathrm{GHz}$.

\subsection{HF-ESR spectroscopy}

\subsubsection{Frequency dependence}

HF-ESR spectra of CuMn2-Green sample at four different frequencies, together with the corresponding frequency vs. resonance magnetic field diagram $\left(\nu\right.$ vs. $\left.H_{r e s}\right)$, are presented in Fig. 3 . Here, four resonance branches (corresponding to the four observed ESR lines) are obtained as linear fits through experimental points (symbols). From the slopes of the resonance branches, the $g$-factor could be determined as $g=\left(h / \mu_{B}\right) \partial \nu / \partial H_{\text {res }}$ 34, where $h$ is the Planck constant and $\mu_{B}$ is the Bohr magneton. In this way, four different values of the $g$-factor were obtained. Two sharp ESR lines with $g_{1} \approx 2.00$ and $g_{2} \approx 1.99$ are assigned to $\mathrm{Mn}(\mathrm{II})$ ions (to be further discussed in Section 4); while the other two lines, $g_{\|} \approx 2.26$ and $g_{\perp} \approx 2.06$, correspond to the parallel and perpendicular $g$-factors of $\mathrm{Cu}(\mathrm{II})$ ions, respectively. 35 ] 


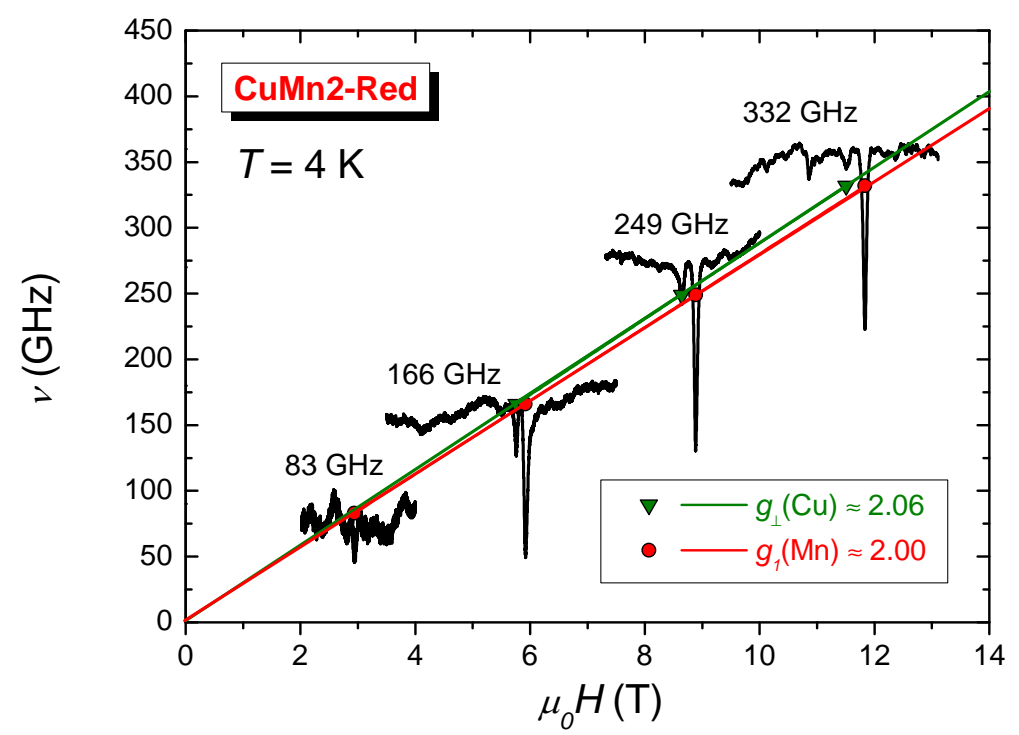

Figure 4: Frequency $\nu$ vs. resonance magnetic field $\mu_{0} H$ dependence (symbols), together with corresponding spectra, at $T=4 \mathrm{~K}$. Solid lines are linear fits to the experimental data (symbols).

HF-ESR spectra of CuMn2-Red sample at four different frequencies and the $\nu$ vs. $H_{\text {res }}$ diagram, are presented in Fig. 4 In contrast to CuMn2-Green, here, we observe only one sharp ESR line with $g_{1} \approx 2.00$ corresponding to $\mathrm{Mn}(\mathrm{II})$ ions. The second observed line with $g_{\perp} \approx 2.06$, similar to CuMn2-Green, corresponds to the perpendicular $g$-factor of $\mathrm{Cu}$ (II) ions. The contribution of the parallel orientation of $\mathrm{Cu}$ (II) ions was not detected due to a small amount of the sample resulting in a weak signal intensity. It should be mentioned that a large number of experiments was performed and only one sharp $\mathrm{Mn}$ (II) line was always detected in the CuMn2-Red spectra.

Fig. 5 shows the HF-ESR spectra and the $\nu$ vs. $H_{\text {res }}$ diagram for CoMn2Yellow. Three resonance branches (corresponding to three observed ESR lines) give three different $g$-factor values. Here, we again see two sharp ESR lines with $g_{1} \approx 2.00$ and $g_{2} \approx 1.99$ which correspond to $\mathrm{Mn}(\mathrm{II})$ ions. Note, the two $\mathrm{Mn}$ (II) 


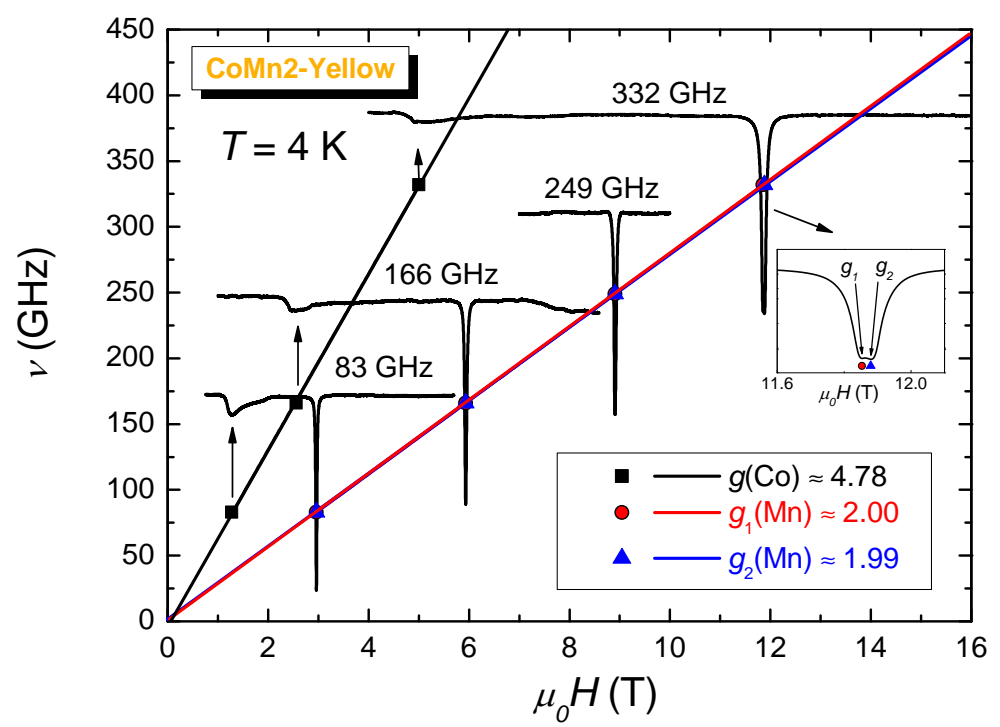

Figure 5: Frequency $\nu$ vs. resonance magnetic field $\mu_{0} H$ dependence (symbols), together with corresponding spectra, at $T=4 \mathrm{~K}$. Solid lines are linear fits to the experimental data (symbols). Inset: Enlarged central part of the spectra at $332 \mathrm{GHz}$.

lines could be resolved only at the highest excitation frequency of $332 \mathrm{GHz}$. The broad line with $g \approx 4.78$ is associated with $\mathrm{Co}(\mathrm{II})$ ions.

The extrapolation of the resonance branches to the axis $H=0$ shows zero magnetic anisotropy gap for all detected ESR lines in all three studied samples, i.e., all branches go through the origin $\left(\mu_{0} H, \nu\right)=(0,0)$. Absence of magnetic anisotropy is expected for $\mathrm{Cu}(\mathrm{II})$ ions due to the Kramers degeneracy of the spin $S=1 / 2$. For $\operatorname{Mn}(\mathrm{II})$ ions, the non-detectable zero-field splitting is in agreement with approximately octahedral environment [36] (as could be seen from crystallographic data). Fine structure typically observed for single Mn(II) ions is not visible here suggesting a sizable exchange interaction between $\mathrm{Mn}$ (II) spin centers that is responsible for a collapse of the fine structure into a single line (exchange narrowing). For high-spin $\mathrm{Co}$ (II) ions with spin $S=3 / 2,10$ we assume that magnetic anisotropy is very large and therefore, only the lowest 

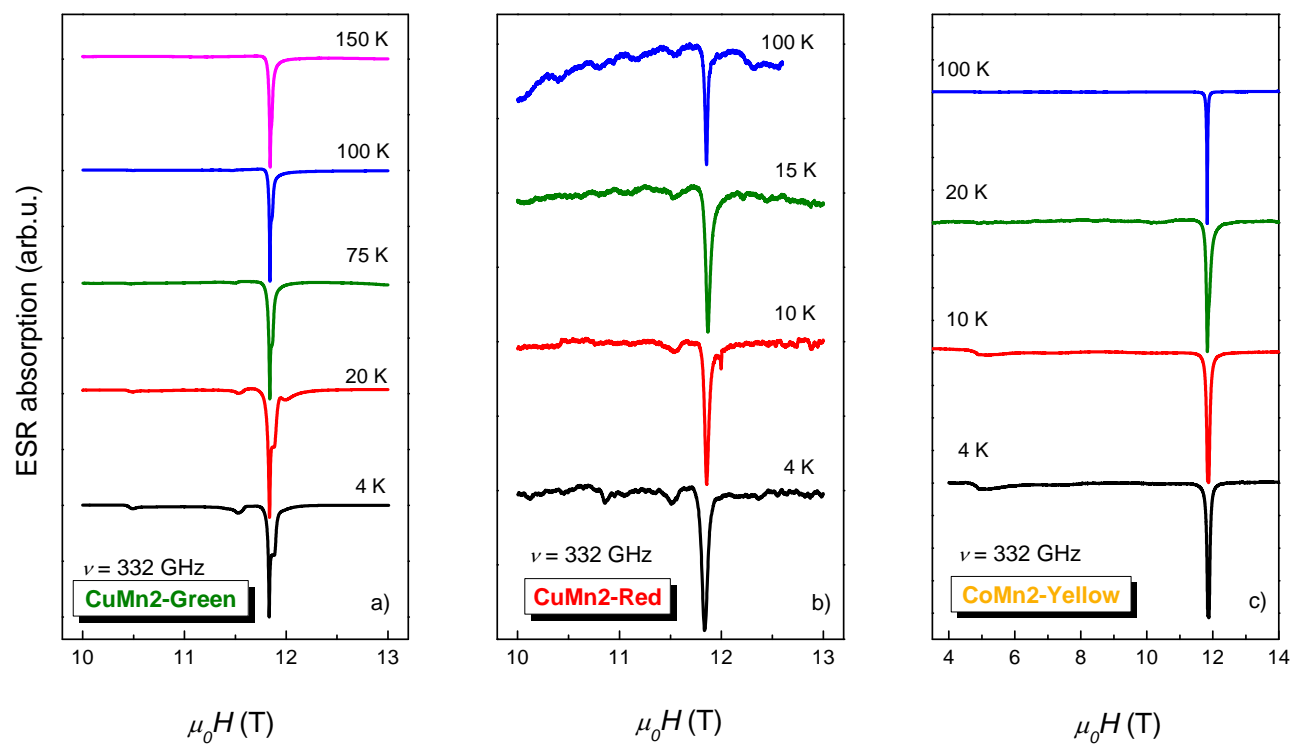

Figure 6: Temperature dependence of ESR spectra of a) CuMn2-Green, b) CuMn2-Red and c) CoMn2-Yellow sample at frequency $\nu=332 \mathrm{GHz}$. Intensities of the lines are scaled to have same heights of the central $\mathrm{Mn}(\mathrm{II})$ peak, for the each compound.

Kramers doublet $(+1 / 2$ and $-1 / 2)$ is thermally populated resulting in a gapless ESR transition. [36, 37].

\subsubsection{Temperature dependence}

Temperature dependence of the HF-ESR spectra of all three samples measured at the highest applied frequency $332 \mathrm{GHz}$ is presented in Fig. 6.

These measurements revealed the presence of sharp ESR lines corresponding to the $\mathrm{Mn}(\mathrm{II})$ ions in all three samples at all investigated temperatures. In contrast, X-band ESR measurements (see Fig. 7 in Ref. [11] and Suppl. Figs. 4 and 5) showed broadening and disappearing of the $\mathrm{Mn}(\mathrm{II})$ line at low temperatures ${ }_{230}(12-13 \mathrm{~K})$ due to antiferromagnetic correlations between the Mn spins. 


\subsection{Simulation}

To obtain more accurate values of the g-tensor for the studied compounds, simulation of the ESR spectra was performed using the EasySpin software [38. For the simulation we used the reduced form of the spin-Hamiltonian for all $\mathrm{Mn}(\mathrm{II})$ and $\mathrm{Co}$ (II) ions, respectively, that only roughly agrees with the values obtained from ESR (Table 2).

\section{Discussion}

The magnetic ground state of the studied compounds could be described as an AFM network of slightly canted Mn(II) spins with the contribution of paramagnetic $\mathrm{Cu}(\mathrm{II})$ ions in CuMn2-Green and CuMn2-Red samples and the contribution of $\mathrm{Co}$ (II), including non-zero spin-orbit coupling, in CoMn2-Yellow 


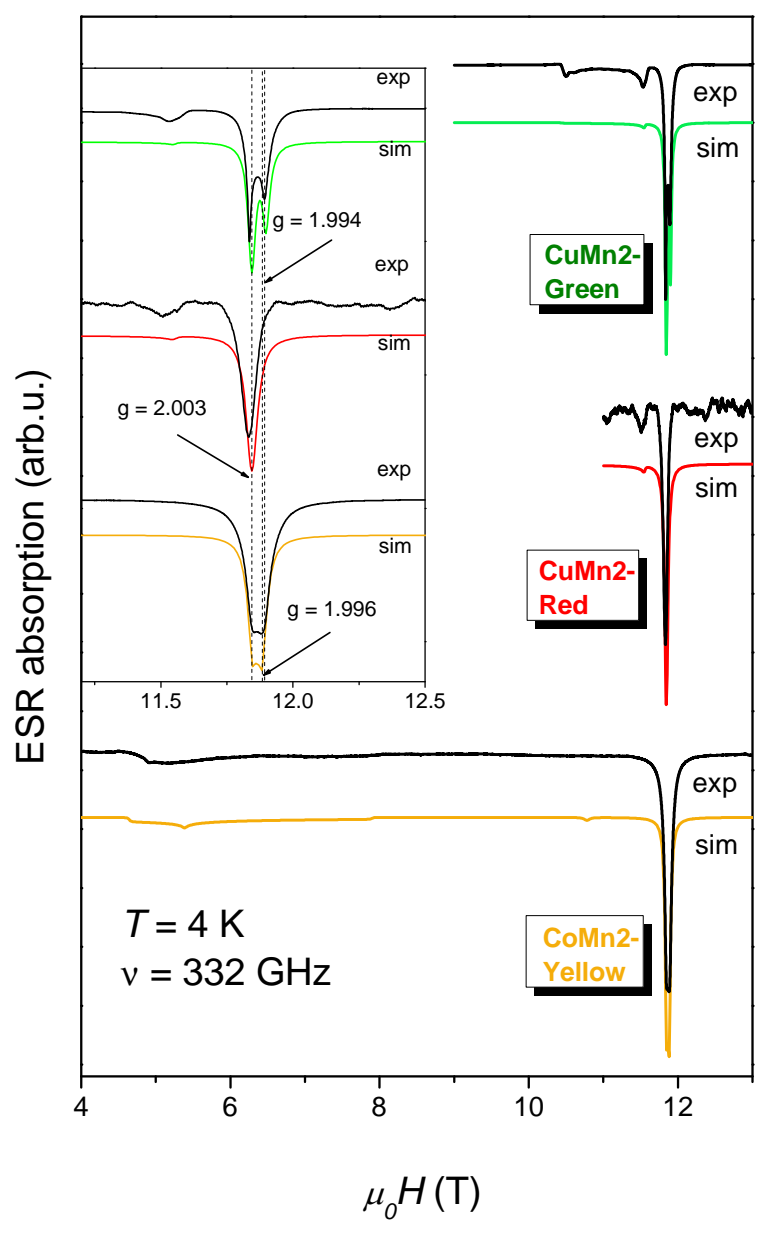

Figure 7: Experimental and simulated ESR spectra of CuMn2-Green, CuMn2-Red and CoMn2-Yellow, obtained at $T=4 \mathrm{~K}$ and frequency $\nu=332 \mathrm{GHz}$. Inset: Enlarged spectra around $g \approx 2$; the splitting of the $\mathrm{Mn}$ (II) lines is clearly visible. 
Table 2: The $\mathbf{g}$ tensor parameters of CuMn2-Green, CuMn2-Red and CoMn2-Yellow samples.

\begin{tabular}{|c|c|c|c|c|c|c|c|}
\hline & \multicolumn{2}{|l|}{$\mathrm{Cu}(\mathrm{II})$} & \multicolumn{2}{|l|}{$\mathrm{Mn}(\mathrm{II})$} & \multicolumn{3}{|c|}{$\mathrm{Co}(\mathrm{II})$} \\
\hline & $g_{\perp}$ & $g_{\|}$ & $g_{1}$ & $g_{2}$ & $g_{x}$ & $g_{y}$ & $g_{z}$ \\
\hline CuMn2-Green & 2.053 & 2.27 & 2.003 & 1.994 & - & - & - \\
\hline CuMn2-Red & 2.053 & 2.27 & 2.003 & - & - & - & - \\
\hline CoMn2-Yellow & - & - & 2.003 & 1.996 & 5.1 & 4.4 & 3.0 \\
\hline
\end{tabular}

sample [10, 11]. The magnetic ordering in these $\mathrm{Mn}(\mathrm{II})$ networks was studied by magnetic susceptibility [39, 16, 10, 11], Mössbauer spectroscopy [39] and neutron diffraction[14 measurements but yet is not well understood. The weak canting of the magnetic moments in the AFM phase could be connected to occurrence of an antisymmetric Dzyaloshinsky-Moriya interaction, allowed by the non-centrosymmetric space group of these samples.

The temperature at which the manganese line disappears in X-band ESR spectra (Suppl. Fig. 5 and Fig. 7 in Ref. [11] for CoMn2-Yellow and CuMn2Green, respectively) coincides with the intersection of the zero-field cooled (ZFS) and field-cooled (FC) curves obtained by magnetization measurements [10, 11]. This temperature $T=12-13 \mathrm{~K}$ corresponds to the temperature of the phase transition of the $\mathrm{Mn}(\mathrm{II})$ oxalate-network into an ordered AFM state and it is in agreement with the Néel temperature found for Mn(II) oxalate-networks in similar compounds [Fe(bpy) $\left.)_{3}\right]\left[\mathrm{Mn}_{2}\left(\mathrm{C}_{2} \mathrm{O}_{4}\right)_{3}\right]$ [39, 14] and $\left[\mathrm{Ru}(\mathrm{bpy})_{3}\right]\left[\mathrm{Mn}_{2}\left(\mathrm{C}_{2} \mathrm{O}_{4}\right)_{3}\right]$ [16. While the phase transition could be observed in X-band ESR spectra as disappearance of the $\mathrm{Mn}$ (II) line, this line remains unchanged in HF-ESR spectra measured down to $4 \mathrm{~K}$, suggesting the absence of the magnetic phase transition. Obviously, high magnetic fields (above $1 \mathrm{~T}$ ) suppress the antiferromagnetic ordering in these systems in agreement with magnetization measurements[10].

Besides magnetic ordering, an interesting point is related to the colors of these $\mathrm{Mn}(\mathrm{II})$ oxalate-based magnets, namely, why the investigated compounds $\left\{\left[\mathrm{Cu}(\text { bpy })_{3}\right]\left[\mathrm{Mn}_{2}\left(\mathrm{C}_{2} \mathrm{O}_{4}\right)_{3}\right] \cdot \mathrm{H}_{2} \mathrm{O}\right\}_{n}$ have different colors. It is well known, that 280 structural defects in the crystal lattice can cause changes in physical properties. 
For example surface defects due to different single crystal sizes and surface to volume ratios could play a role. 40. Indeed, CuMn2-Red crystals were few times smaller compared to CuMn2-Green crystals, as could be seen in the Suppl. Fig. 1. However, polycrystalline samples ground into fine powders maintained the same 285 colors as the single crystals and therefore, crystal size effect could be excluded.

Another aspect that could influence the color of the studied compounds is the composition. However here, the EDX analysis showed that the average $\mathrm{Mn}$ to $\mathrm{Cu}$ ratio is $2.02 \pm 0.04$ and $1.97 \pm 0.02$ in CuMn2-Green and CuMn2Red sample, respectively (Suppl. Table 2), that is approximately the same ratio within the error bars. This does not exclude the possibility that some manganese ions are occupy copper sites or vice versa. In that case, ESR signal of $\mathrm{Cu}(\mathrm{II})$ ions placed on $\mathrm{Mn}(\mathrm{II})$ sites could not been observed due to the strong exchange interaction in the oxalate-network. On the other hand, if there is some amount of $\mathrm{Mn}(\mathrm{II})$ ions $(\sim 1 \%)$ placed instead of regular $\mathrm{Cu}(\mathrm{II})$ ions in the vacancies of the oxalate-network, its contribution should be paramagnetic and visible in the ESR spectrum as 6 hyperfine-splitted lines centered at $g=2$ [41, which is not the case.

It is known that high-spin $\mathrm{Mn}(\mathrm{II})$ complexes in octahedral configuration have spin forbidden $(\Delta S \neq 0)$ as well as parity forbidden (the Laporte rule) transitions in electronic spectra, which results in the pale pink color of these compounds [42, 43]. In tetrahedral environment, the transitions are still spin forbidden but no longer parity forbidden and therefore are $\sim 100$ times stronger. Such compounds have noticeable colors 42 which is also the case for the studied compounds (see Suppl. Fig. 7), as well as for similar compounds [Fe(bpy) 3 ]$\left[\mathrm{Mn}_{2}\left(\mathrm{C}_{2} \mathrm{O}_{4}\right)_{3}\right]\left[39\right.$ and $\left[\mathrm{Ru}(\text { bpy })_{3}\right]\left[\mathrm{Mn}_{2}\left(\mathrm{C}_{2} \mathrm{O}_{4}\right)_{3}\right][16$ (both are red). Thus, the difference in $\mathrm{Mn}(\mathrm{II})$ oxalate-networks, due to different ligand field distortion around $\mathrm{Mn}(\mathrm{II})$ centers can change electronic transitions in the optical spectra and therefore cause different colors [42, 43].

HF-ESR spectra of CuMn2-Green (Figs. 3, 6, 7) clearly show two close lines 310 with $g_{1}=2.003$ and $g_{2}=1.994$ (Table 2 ) which both could be assigned to $\mathrm{Mn}$ (II) ions, placed in the oxalate-network. Similar two lines with values $g_{1}=2.003$ 
and $g_{2}=1.996$ are observed also for CoMn2-Yellow sample. The value of $g$ that is a little bit smaller compared to the free spin value of 2.00232 is expected for the ions with a half-filled shell. 35. Two observed $g$ values are very close and could not be resolved by the usually used characterization techniques, such as magnetization measurements and X-band ESR spectroscopy [11, 10, 44, 21]. However, using high excitation frequencies and high magnetic fields, it is possible to resolve such a small difference in $g$ values. It is known that distortion of $\mathrm{Mn}(\mathrm{II})$ octahedra produces anisotropy of $g$-factor and therefore could cause lineshape changes. 45. However, here observed CuMn2-Green spectra do not have a typical anisotropic powder lineshape, characterized with a peak and a shoulder. Two observed $g$-factors point to two slightly different types of Mn(II) centers due to slight variation of the local ligand coordinations. Thus the observation of two ESR lines indicates the two different $\mathrm{Mn}(\mathrm{II})$ centers, possibly related to racemic conglomerate structure with two enantiomers in the investigated sample. The HF-ESR results have shown that, despite the same X-ray parameters of CuMn2-Green and CuMn2-Red compounds, Mn(II) ions are not absolutely equivalent in their oxalate-networks. This difference in the coordination environment could cause the difference in the color of the two compounds.

\section{Conclusion}

Here, we have presented the detailed HF-ESR study of three 3D oxalatebased coordination polymers, supported by crystallographic, photoluminescence, EDX and X-band ESR analysis. HF-ESR measurements have shown that the 335 AFM ordering (below $T_{N} \approx 13 \mathrm{~K}$ ) in these compounds is suppressed by high magnetic fields $(>1 \mathrm{~T})$, in agreement with magnetic susceptibility measurements. [10, 11] Moreover, from these measurements we have determined the parameters of the g-tensor for all three metal ions: $\mathrm{Cu}(\mathrm{II}), \mathrm{Co}(\mathrm{II})$ and $\mathrm{Mn}(\mathrm{II})$.

The central observation in this study is the detection of two different ESR lines (with different $g$-factors) in the spectra of CuMn2-Green and CoMn2- 
Yellow while for CuMn2-Red only one ESR line is observed. This reveals the presence of two slightly different $\mathrm{Mn}$ (II) centers in CuMn2-Green and CoMn2Yellow, that is beyond the detection limit of other usually used characterization techniques such as X-ray diffraction, magnetic susceptibility measurement and

345 X-, Q- or W-band ESR. This small difference in the local environment of $\mathrm{Mn}(\mathrm{II})$ ions in the oxalate-networks of CuMn2-Green compared to CuMn2-Red, visible only due to the excellent resolution of HF-ESR, could be related with different colors of the investigated coordination polymers.

\section{Appendix A. Supplementary data}

CCDC 1504116 contains the supplementary crystallographic data for CuMn2-

Red. These data can be obtained free of charge via http://www.ccdc.cam.ac.uk/conts/retrieving.html, or from the Cambridge Crystallographic Data Centre, 12 Union Road, Cambridge CB2 1EZ, UK; fax: (+44) 1223-336-033; or e-mail: deposit@ccdc.cam.ac.uk.

\section{Acknowledgements}

The work of D. Žilić at the IFW Dresden was realized through the Croatian Science Foundation (HRZZ) postdoc scholarship (project 02.03/164). This research was supported in part by the HRZZ projects 1108 and IP-2014-094079. The work was supported in part by the Deutsche Forschungsgemeinschaft through FOR1154 "Towards Molecular Spintronics". The authors are grateful to Jasna Dasović from RBI-Zagreb for recording PL spectra.

\section{References}

[1] S. R. Batten, N. R. Champness, X.-M. Chen, J. Garcia-Martinez, S. Kitagawa, L. Ohrstrom, M. O'Keeffe, M. P. Suh, J. Reedijk, CrystEngComm 14 (2012) 3001-3004.

365

[2] H.-C. Zhou, J. R. Long, O. M. Yaghi, Chem. Rev. 112 (2) (2012) 673-674.

[3] S. L. James, Chem. Soc. Rev. 32 (2003) 276-288. 
[4] S. Kitagawa, R. Kitaura, S.-i. Noro, Angew. Chem. Int. Ed. 43 (2004) 2334-2375.

[5] C. N. R. Rao, S. Natarajan, R. Vaidhyanathan, Angew. Chem. Int. Ed. 43 (2004) 1466-1496.

[6] M. P. Suh, Y. E. Cheon, E. Y. Lee, Coord. Chem. Rev. 252 (2008) 10071026.

[7] R. J. Kuppler, D. J. Timmons, Q.-R. Fang, J.-R. Li, T. A. Makal, M. D. Young, D. Yuan, D. Zhao, W. Zhuang, H.-C. Zhou, Coord. Chem. Rev. 253 (2009) 3042-3066.

[8] T. Yamada, K. Otsubo, R. Makiura, H. Kitagawa, Chem. Soc. Rev. 42 (2013) 6655-6669.

[9] G. Marinescu, M. Andruh, F. Lloret, M. Julve, Coord. Chem. Rev. 255 (2011) 161-185.

[10] J. Habjanič, M. Jurić, J. Popović, K. Molčanov, D. Pajić, Inorg. Chem. 53 (2014) 9633-9643.

[11] M. Jurić, D. Pajić, D. Žilić, B. Rakvin, K. Molčanov, J. Popović, Dalton Trans. 44 (2015) 20626-20635.

[12] E. Coronado, C. Martí-Gastaldo, J. R. Galán-Mascarós, M. Cavallini, J. Am. Chem. Soc. 132 (2010) 5456-5468.

[13] C. Maxim, S. Ferlay, H. Tokoro, S.-I. Ohkoshi, C. Train, Chem. Commun. 50 (2014) 5629-5632.

[14] S. Decurtins, H. W. Schmalle, R. Pellaux, R. Huber, P. Fischer, B. Ouladdiaf, Adv. Mater. 8 (8) (1996) 647-651.

390 [15] S. Chorazy, K. Nakabayashi, S. Ohkoshi, B. Sieklucka, Chem. Mater. 26 (2014) 4072-4075. 
[16] F. Pointillart, C. Train, K. Boubekeur, M. Gruselle, M. Verdaguer, Tetrahedron: Asymmetry 17 (2006) 1937 - 1943.

[17] A.-L. Barra, D. Gatteschi, R. Sessoli, L. Sorace, Magn. Res. Chem. 43 (2005) S183-S191.

[18] Y. Krupskaya, A. Alfonsov, A. Parameswaran, V. Kataev, R. Klingeler, G. Steinfeld, N. Beyer, M. Gressenbuch, B. Kersting, B. Büchner, ChemPhysChem 11 (2010) 1961-1970.

[19] A. Das, K. Gieb, Y. Krupskaya, S. Demeshko, S. Dechert, R. Klingeler, V. Kataev, B. Büchner, P. Müller, F. Meyer, J. Am. Chem. Soc. 133 (2011) 3433-3443.

[20] Z. Wang, J. van Tol, T. Taguchi, M. R. Daniels, G. Christou, N. S. Dalal, JACS 133 (44) (2011) 17586-17589.

[21] D. Žilić, L. Androš, Y. Krupskaya, V. Kataev, B. Büchner, Appl. Magn. Reson. 46 (2015) 309-321.

[22] G. H. Cartledge, W. P. Ericks, J. Am. Chem. Soc. 58 (1936) 2061-2065.

[23] F. M. Jaeger, J. A. Dijk, Z. Anorg. Allg. Chem. 227 (1936) 273-327.

[24] CrysAlis PRO, Oxford Diffraction Ltd., U. K., 2007.

[25] G. M. Sheldrick, Acta Crystallogr., Sect. A 64 (2008) 112-122.

[26] A. L. Spek, Acta Crystallogr., Sect. D 65 (2009) 148-155.

[27] L. J. Farrugia, J. Appl. Crystallogr. 45 (4) (2012) 849-854.

[28] C. F. Macrae, I. J. Bruno, J. A. Chisholm, P. R. Edgington, P. McCabe, E. Pidcock, L. Rodriguez-Monge, R. Taylor, J. Van De Streek, P. A. Wood, J. Appl. Crystallogr. 41 (2) (2008) 466-470.

${ }_{415}$ [29] C. Golze, A. Alfonsov, R. Klingeler, B. Büchner, V. Kataev, C. Mennerich, H.-H. Klauss, M. Goiran, J.-M. Broto, H. Rakoto, S. Demeshko, G. Leibeling, F. Meyer, Phys. Rev. B 73 (2006) 224403. 
[30] B. Spingler, S. Schnidrig, T. Todorova, F. Wild, CrystEngComm 14 (2012) $751-757$.

420

[32] S. Hyde, M. O’Keeffe, D. M. Proserpio, Angew. Chem. Int. Ed. 47 (2008) $7996-8000$.

[33] R. Andrés, M. Brissard, M. Gruselle, C. Train, J. Vaissermann,

[36] A. Carrington, M. A. D., Introduction to Magnetic Resonance, Harper and Row, New York, 1967.

[37] O. Kahn, Molecular Magnetism, Wiley-VCH Inc., 1993.

[38] S. Stoll, A. Schweiger, J. Magn. Reson. 178 (2006) 42-55.

${ }_{435}$ [39] S. Decurtins, H. W. Schmalle, P. Schneuwly, J. Ensling, P. Gütlich, J. Am. Chem. Soc. 116 (1994) 9521-9528.

[40] C. Karunatilaka, D.-K. Bučar, L. R. Ditzler, T. Friščić, D. C. Swenson, L. R. MacGillivray, A. V. Tivanski, Angew. Chem. Int. Ed. 50 (2011) 86428646.

440

[41] D. M. L. Goodgame, H. E. Mkami, G. M. Smith, J. P. Zhao, E. J. L. McInnes, Dalton Trans. (2003) 34-35.

[42] F. A. Cotton, G. Wilkinson, Advanced Inorganic Chemistry: a Comprehensive Text, 5th Edition, Wiley, New York, 1988. 
[43] B. N. Figgis, M. A. Hitchman, Ligand Field Theory and Its Applications, Wiley-VCH, New York, 2000.

[44] A. Bencini, D. Gatteschi, Electron Paramagnetic Resonance of Exchange Coupled Systems, Springer-Verlag, Berlin Heidelberg, 1990.

[45] Y. Lei, X. Zu, M. Zhao, Physica B 364 (2005) 122-129. 